\title{
The chemistry of etching and deposition processes
}

\author{
B. d'Agostino, F. Fracassi, R. Lamendola
}

Centro di Studio per la Chimica dei Plasmi, C.N.R, Department of Chemistry, University of Bari, via Orabona 4, 70126 Bari, Italy

Abstract: XPS surface analysis, particularly with an in situ approach, coupled with plasma diagnostic techniques, gives a fairly good insight of plasma-surface interactions. Specific examples to be discussed include etching of $\mathrm{Al}, \mathrm{Ti}, \mathrm{TiN}, \mathrm{W}$, and deposition of thin films from organosilicon precursors.

\section{INTRODUCTION}

The great deal of work performed in the 80 's in the field of plasma processing (see for example ref. $1-5$ and references therein) has produced at least two important results: (a) an impressive growth of utilization of plasma technology for many update applications, and (b) a generalized minimum understanding of the microscopic mechanism of interaction, particularly for the etching processes. The former point is witnessed by the relevance of novel applications for biomaterials, food packaging, tool hardening, microelectronics, optics and optoelectronics, etc. The latter issue has generated a noticeable decrease, in the last few years, of fundamental work addressed to the understanding of plasma-surface interaction mechanism. This is particularly true in the field of plasma etching, where the role of the main active species and of charged particles seems to be ruled for most important cases. A long time has elapsed since August 1984 when an exciting workshop was chaired by D. L. Flamm during the Gordon Research Conference on Plasma Chemistry devoted to the role of F-atoms in silicon etching (Note a). For the plasma enhanced chemical vapor deposition the situation is different, particularly in those fields which are not pushed by microelectronic industry. It seems, at least to these authors, that in this case a more empiric and utilization-aimed work is produced, mostly with the trial-and-error approach.

It is useless to say that the best improvements in every field can be obtained only if a complete understanding of the reaction mechanism and of the effect of process variables is achieved. This is an ambitious goal which can be reached, case by case, by selecting the proper experiments and the right diagnostic techniques. The aim should be to control each plasma variable (e.g. ion

Note a: Gordon Research Conference on Plasma Chemistry, T.A. Miller Chairman, Tilton, 1984. 
bombardment energy, substrate temperature, active species concentration, etc.) independently from the others and to have a clear picture of surface conditions during the treatment. It is our opinion, therefore, that both plasma etching and polymer deposition would significantly benefit from in situ study of surface composition during the plasma process. Bulk materials, in fact, communicate with gas-phase active species and precursors through a surface. Since the plasma medium is not compatible with delicate surface analysis apparatuses two technical solutions are currently reported in the literature for in situ surface investigation: a) the samples are treated in a plasma reactor and transferred under vacuum (in order to avoid surface modifications and contamination by air exposure) in a surface analysis apparatus (XPS, AUGER, etc.); b) the experiments are performed in sophisticated plasmaless apparatuses in ultra high vacuum conditions which simulate the plasma with ion, radical and molecular beams but which are compatible with surface analysis apparatuses. Both approaches are valid and have advantages and disadvantages. The former, which has been utilized to reach part of the conclusions reported in this presentation, allows to obtain information in real conditions, at the pressure currently utilized during the process under study, in the presence of the same contaminant species. This is an important issue, it goes without saying, in fact, that the extent of surface contaminations, the presence of inhibitors or barrier layers are all parameters markedly affecting the adsorption of gaseous components, process kinetics, material properties and process performances. Of course, in order to consider the analysis as an in situ technique, it must be assumed that the sample surface is stable and does not change during the time elapsed between the end of the plasma treatment and the analysis. A typical example of a plasma reactor connected by means of a high vacuum transfer arm (10-8 torr) to a surface diagnostic apparatus (i.e. $X$ ray photoelectron spectroscopy system (XPS)) is reported in Fig. 1 (6). The system allows to analyze samples $10 \mathrm{~cm}$ in diameter without external contaminations.

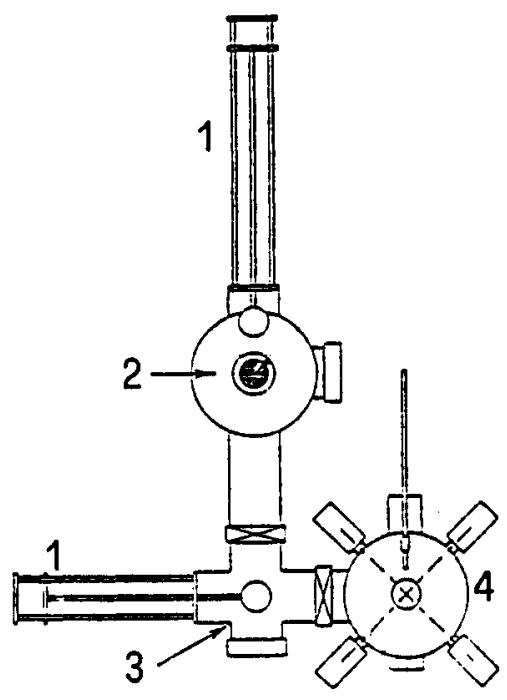

Fig. 1. Plasma reactor connected under vacuum ta an XPS apparatus. 1) manipulator, 2) plasma reactor, 3) high vacuum buffer chamber, 4) XPS chamber.

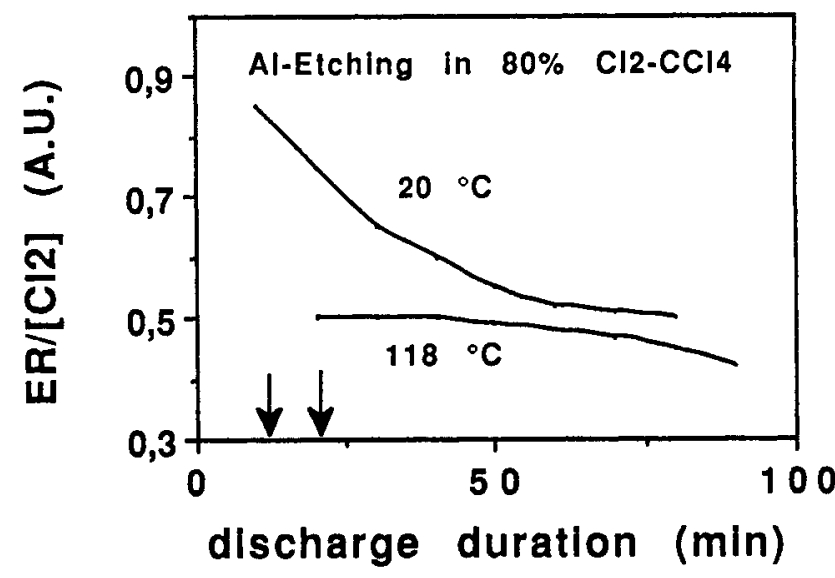

Fig. 2. $\mathrm{ER} /\left[\mathrm{Cl}_{2}\right]$ vs. time elapsed since discharge is turned on for $A l$ etching at two substrate temperatures. The arrows indicate the induction time 
In this presentation we discuss some important issues of plasma etching and deposition (from organosilicon compounds) with particular attention to the surface conditions and with some examples of in situ surface diagnostics.

\section{PLASMA ASSISTED DRY ETCHING}

Surface contamination plays a very important role in dry etching reactions either because new layers can deposit on the samples or because the structure and chemical composition of existing surfaces can be modified.

If one has to tell the story of contaminants and of non volatile layers in the chemistry of etching processes and in the overall etching kinetics, a fairly good example to start with is that of aluminum etching. Aluminum thin films are always covered by an inert surface layer of $\mathrm{Al}_{2} \mathrm{O}_{3}$, which causes the well known delay between discharge ignition and the etching process start (induction time). To remove the surface contaminant layer strong ion bombardment and/or the appropriate chemistry are required (e.g. $\mathrm{CCl}_{4}$ or $\mathrm{BCl}_{3}$ addition to the feed). When the induction time is over, etching can proceed in chlorine by the reaction of chlorine molecules and/or atoms as a function of the experimental conditions. Fig. 2 shows trends of $\mathrm{Al}$ etch rates divided by chlorine concentration (the active etchant species), $\mathrm{E} /\left[\mathrm{Cl}_{2}\right]$, as a function of the time elapsed since the discharge is turned on, in plasmas fed with $\mathrm{Cl}_{2}-\mathrm{CCl}_{4}$ mixtures at 20 and $118^{\circ} \mathrm{C}$ as substrate temperatures (7). The etching rate decreases because under these conditions a deposit containing the etch products, $\mathrm{AlCl}_{3}$ and/or $\mathrm{Al}_{2} \mathrm{Cl}_{6}$, as well as a chlorocarbon polymer, is formed on the aluminum surface. The thickness of this deposit increases with time particularly at low temperature (film deposition is generally favoured by low temperatures). The contaminant layer does interfere with the etching kinetics since it acts as a barrier to the ingoing etchant species and/or the out-going etch products. At higher temperatures the phenomenon is much reduced because the thickness of the contaminant film becomes very small. This means that what is usually measured is not the real chemical Al-etch rate, that is to say the value in the absence of barrier films of contaminant chlorocarbon polymer and etch products, but an 'apparent' physico-chemical rate which includes through-film diffusion effects. The true chemical etch rate, without the distortions introduced by contaminants, can be obtained by the extrapolations of the curves of Fig. 2 at zero time.

The presence of a contaminant film, called inhibitor or side wall blocking agent, is often required to promote directional etching as in the case of tungsten dry etching in fluorocarbon mixtures. In this case in situ Auger spectroscopy has been utilized to study the effect of low energy ion bombardment on the formation of a fluorocarbon contaminant film in a triodereactor fed with $\mathrm{CF}_{4}-\mathrm{H}_{2}$ (and $\mathrm{CF}_{4}-\mathrm{O}_{2}$ ) (8). It has been shown that on the surfaces interested by ion bombardment the etching process occurs even at high hydrogen concentration, conditions known to be suitable for deposition and not for etching. These surfaces, in fact, are not completely covered by fluoropolymers and bare tungsten is available for reaction with fluorine atoms. On the other hand, surfaces which are not subjected to ion bombardment are covered by a thick film of contaminant and the etching process completely stops.

The formation of contaminant layers is not only a peculiarity of those processes characterized by low volatile etch products and/or polymerizing 
etching mixtures as in the previous examples. During titanium dry etching in $\mathrm{CF}_{4}-\mathrm{O}_{2}$, for instance, the substrate surface is covered by a thick hyperfluorinated layer of titanium fluorides (9), which is able to trap some fluorine $\left(F_{2}\right.$, and/or $\left.F^{-}\right)$. The thickness of this layer strongly depends on the working conditions but it has never been found to be less than 50-60 $\AA$. The presence of trapped species in the surface of etched samples has also been revealed in the case of tungsten etching in fluorine containing plasmas, in this case the surface contains $W F_{4}, W F_{5}$, and as trapped species $W F_{6}$ (the etch product) and $F_{2}$ (or $\left.F^{-}\right)(6,10)$.

In situ diagnostic techniques are a must to give a rationale to the etching processes of extremely reactive surfaces as, for instance, that of titanium and tungsten. Titanium forms a layer of 'hard' quasi -stoichiometric $\mathrm{TiO}_{2}$ over one of a 'soft' $\mathrm{TiO}_{x}(9)$ (the adjectives hard and soft here are used to qualify the extent of reactivity with the etchant species). As in the case of aluminum etching, the hard oxide is responsible for the induction time. In situ XPS analyses, coupled to etch rate measurements, in fact, have shown that the process of titanium etching consists of three successive steps: 1 - the very slow ablation of the 'hard' stoichiometric oxide; 2- the etching of the non stoichiometric $\mathrm{TiO}_{x}$ layer; 3 - the etching of metallic clean titanium. When the oxidized surface layer is etched away and $\mathrm{Ti}$ is exposed to a fluorine containing plasma, its surface is covered with a contaminant fluorinated layer (11) which, for instance, precludes it from utilization as sublayer for tungsten CVD (12). The induction time due to surface oxide has also been detected in the dry etching of titanium nitride, even though in this case the contribution of titanium oxynitride cannot be completely ruled out (11). Fig. 3 shows that since $\mathrm{TiO}_{2}$ (and eventually titanium oxynitride) present on the surface has not completely removed, the etching process of TiN does not start appreciably. The spectrum of Fig. 3b, in fact, shows that during the induction time the predominat features of the Ti2p signal are those of fluorinated oxide or oxynitride. The extent of this contamination is lower than that revealed for $\mathrm{Ti}$ etching because while the surface of $\mathrm{Ti}$ during etching is covered by a contaminant layer which includes $\mathrm{TiF}_{3}, \mathrm{TiF}_{4}$ (the etch product) and $\mathrm{F}_{2}$ (or $\mathrm{F}-$ ), for TiN case only TiF and/or TiNxFy have been detected. When the steady state of the dry etching of metallic titanium is reached in fluorinated plasmas, the stable thick hyperfluorinated surface layer protects the surface from reacting with oxygen (from leaks or added to the feed) and water (from leaks) which could induce surface oxidation and therefore decrease the etching rate and the reproducibility of the etching process. This can be appreciated by the the examination of XPS spectra of Fig. 4 collected at the steady state of $\mathrm{Ti}$ etching in different oxygen containing glow discharges. No important differences exist in the Ti2p XPS signals as well as in the oxygen content of the surface when the feed is varied from $100 \% \mathrm{CF}_{4}$ to $\mathrm{CF}_{4}-80 \% \mathrm{O}_{2}$.

The situation is quite different for titanium etching in a chlorine environment, the process requires ion bombardment and is very sensible to oxygen and water vapor. Etching does not start, even for a clean surface and for a discharge fed with chlorine in mixture with $\mathrm{BCl}_{3}$ or $\mathrm{CCl}_{4}$, unless a marked bias or a fluorine pretreatment is performed (13). When the surface is 'cleaned' with an energetic ion beam ( $3 \mathrm{keV}$ ), it becomes moderately reactive and the reaction with chlorine molecules, without air contamination, leads to the formation of both $\mathrm{TiCl}$ and $\mathrm{TiCl}_{2}$. In this case there is not the hyper-halogenation observed with fluorinated mixtures and no etching occurs. If, however, the hard oxide, $\mathrm{TiO}_{2}$, is removed with a $\mathrm{CF}_{4}$ containing plasma, then etching is performed with chlorine 
and the scenario abruptly changes: chlorine can now even spontaneously (i.e. in the absence of a plasma) perform Ti-etching and at a rate higher than in a fluorine chemistry. This can be considered a case of catalysis in which the fluorine present on the surface allows the chlorination reaction for the etching process to follow a quite different pattern, through the formation of $\mathrm{TiCl}_{x} \mathrm{~F}_{y}$ intermediate compounds (13). The above considerations estabilish a practical example of the contamination chemistry for surface processes: contaminants can behave as etching inhibitors, anysotropy promoters and, as in the present case, as process catalyst. On this topic J.W. Coburn has presented some interesting results in his invited talk at the ISPC-10 of Bochum (14). By using two independently controllable sources of atoms/molecules and an energetic ion beam in an ultrahigh vacuum apparatus the author has reported on the $\mathrm{Cl}_{2}$ enhanced reaction of $F$ atoms with $\mathrm{Si}(111)$, the $\mathrm{H}$ atom enhanced reaction of $\mathrm{F}_{2}$ with $\mathrm{Si}(111)$, as well as the catalitic effect of $\mathrm{Pd}$ in the reaction of $\mathrm{H}_{2}$ with fluorine and chlorine.

Peignon, Cardinaud and Turban have studied the etching mechanism of tungsten thin films in $\mathrm{SF}_{6} / \mathrm{O}_{2}$ with the eperimental apparatus reported in fig. 1 (6). Also in this case they had to deal with a surface contaminant layer $\left(\mathrm{WO}_{3}\right)$ which caused a non reproducible induction time. By coupling XPS measurements to plasma phase optical emission spectroscopy and mass spectrometry these

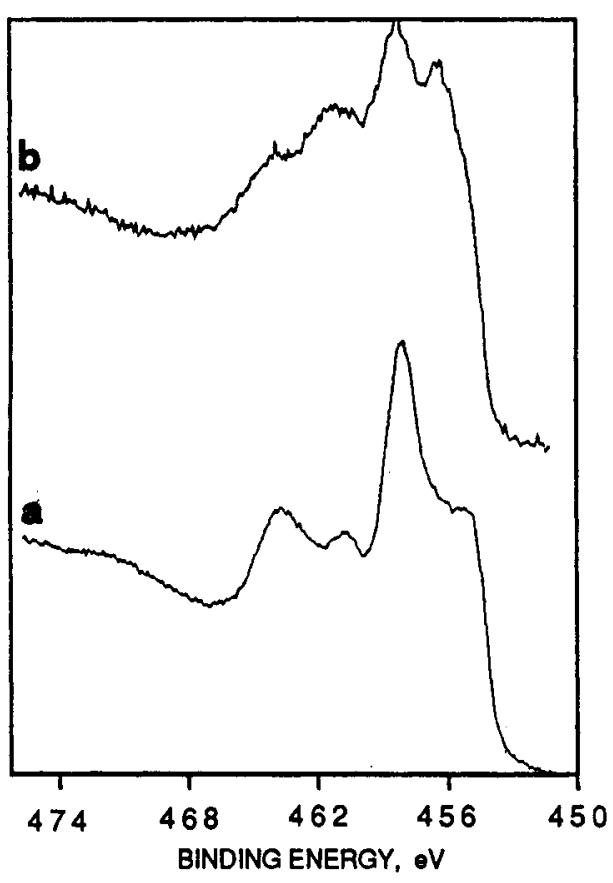

Fig. 3. XPS-Ti2p feature of TiN samples treated in $\mathrm{CF}_{4}$ plasma: a) before the treatment, b) during the induction time (samples analyzed without air exposure).

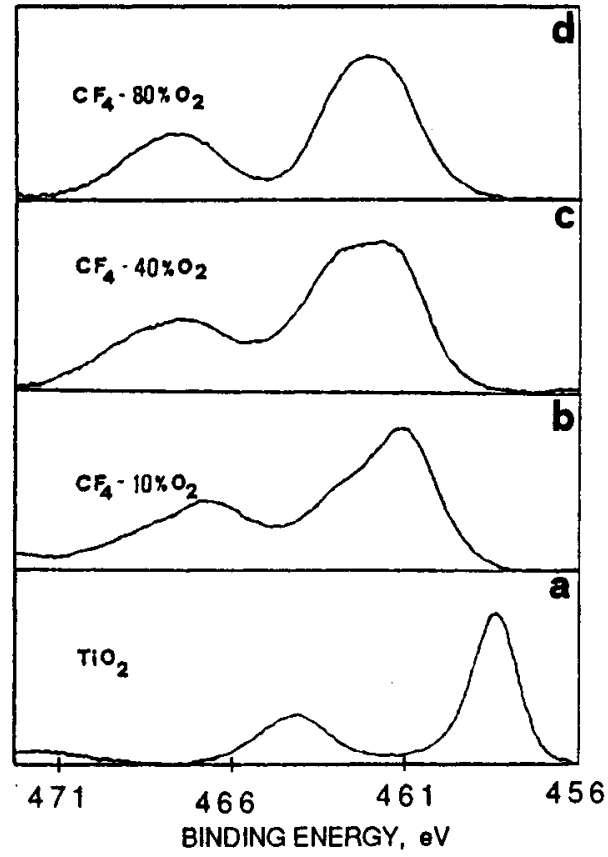

Fig. 4. XPS-Ti2p feature of a untreated $\mathrm{Ti}$ sample $\left(\mathrm{TiO}_{2}\right)$ and of samples exposed for 5 minutes to plasmas of different composition (samples analyzed without air exposure). 
authors focused the attention on the removal of this oxidized layer and showed that either for pure $\mathrm{SF}_{6}$ and $\mathrm{SF}_{6}-\mathrm{O}_{2}$ the etching process consists at least of two steps: a) a rapid transformation of the native oxide $\mathrm{WO}_{3}$ in a fluorinated layer of $W O_{x} F_{y}$ and $\mathrm{SO}_{x}$ and $b$ ) the establishment of a stable etching regime of the unoxidized metal. In the first step the etching product is only $W_{6}$ while, after the surface oxide has been removed and if oxygen is contained in the discharge, also $\mathrm{WOF}_{4}$ can be obtained as etching product. The $\mathrm{WO}_{\mathrm{x}} \mathrm{F}_{\mathrm{y}}$ layer formed in the induction period, therefore, does not have the right chemical structure to generate $\mathrm{WOF}_{4}$.

\section{PLASMA ENHANCED CHEMICAL VAPOR DEPOSITION FROM ORGANOSILICON PRECURSORS}

Silicon containing monomers are often utilized in mixture with oxygen or $\mathrm{N}_{2} \mathrm{O}$ to deposit dielectric $\mathrm{SiO}_{2}$ thin films for microelectronics applications. The main advantages in working with this class of precursors are their low toxicity, the easy handling procedure, particularly if compared to other dangerous compounds such as $\mathrm{SiH}_{4}$, and the better step coverage which can be obtained. To meet the requirements imposed by microelectronic applications, films must have good electrical and mechanical properties. Therefore the deposition must be performed at high temperature $\left(T>250^{\circ} \mathrm{C}\right)$ in order to keep the carbon and water (or $\mathrm{OH}$ group) content as low as possible and to have a stoichiometric $\mathrm{SiO}_{2}$ film. On the other hand, if the deposition is performed at low temperature $\left(T<100^{\circ} \mathrm{C}\right)$ the deposits can be utilized for the surface modification of materials which are not stable at high temperature (plastics), for those applications which do not strictly require good quality $\mathrm{SiO}_{2}$, such as the improvement of surface hardness and of gas barrier properties, biomedical devices, etc. An increasing fraction of PE-CVD studies is devoted to these novel applications. This section of the note reports on the deposition process under these conditions. In fact, the deposition mechanism at high temperatures, which has been studied by several authors (see for instance ref. 15-17), can hardly be extended at low temperatures, either because the experimental conditions are very different and because the etherogeneous gas-surface interactions, which at high temperature play a very important role, are expected to be less important.

The mechanism of a low-temperature deposition process is composed by several steps, whose relative importance depends on the experimental conditions and on the particular film precursors utilized. A schematic block diagram of the deposition mechanism is reported below:

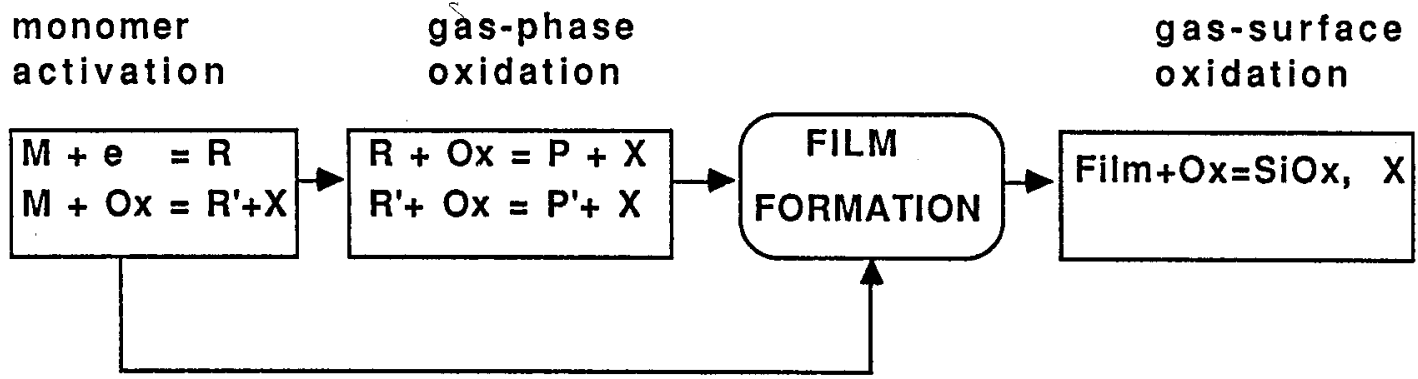


Low-temperature depositions are performed by feeding an organosilicon "monomer" (alone or with a carrier and/or oxidant species) directly in the plasma in order to obtain high monomer activation (high film precursor concentration) and, eventually, high molecular fragmentation. The activation can occur by means of electron collisions and reactions with oxygen atoms or excited molecules $(O x)$.

In this last case partially or totally oxidized compounds $(X)$ are also formed and the carbon and hydrogen content of the film precursors is lowered. The situation can be well appreciated by looking at Fig. 5, where the monomer conversion calculated by mass $\mathrm{sp}$ ectrometry is compared for plasmas fed with Teraethoxysilane (TEOS), Hexamethylsisilazane (HMDSN), and Tetramethylsilane (TMS), in different experimental conditions. What is worthy to stress is that the importance of oxygen activation can be ranked in the following order: curve $1,2,3$. In curve 1 the main channel of activation is electron impact at both low and probably high oxygen-to-monomer ratio of the feed. On the other hand in curves 2 and 3 even a small amount of oxygen is able to increase the monomer activation. The difference in the conversion trends of the three curves of Fig. 5 depends on the different deposition conditions utilized for the experiments (i.e. the residence time).

Mass spectroscopic investigations have shown that plasma fragmentation produces many radicals able to form deposits ( $R$ and $\left.R^{\prime}\right)$; in the case of TEOS it has been reported (18-19) that for mass pattern the main effect of plasma superimposition is the decrease of intensity of peaks derived by TEOS fragmentation pattern, and a marked increase of the intensity of fragments at low $\mathrm{m} / \mathrm{e}$ (e.g. $\mathrm{CH}, \mathrm{CH}_{2}, \mathrm{CH}_{3}$, alcohols, etc.), which corresponds to a strong fragmentation of the organosilicon molecules. Similar results have been obtained for TMS and HMDSN (20). The strong fragmentation allows to rule out, as main deposition pattern, classical chain polymerization mechanisms of reactive intermediates (which, on the other hand, does not take into account the contribution of positive ion bombardment). The overall organosilicon deposition mechanism is complex and many different silicon containing radicals, as well as hydrocarbon radicals, do contribute to the film growth. As it has been demonstrated (21), in fact, hydrocarbon and alcohol radicals are polymerizing species which increase the carbon content of the film. The strong fragmentation of organosilicon compounds utilized as silicon sources is confirmed both by in situ-XPS and by FT-IR analyses $(18,22)$. In situ -XPS investigation is necessary because the surface composition of these deposits, sometime very reactive with oxygen and water, can change after air exposure $(18,23)$. The chemical structure of the film is very different from that of the starting compound and the process occurs with practically no structure retention. In Fig. 6 the FT-IR spectrum of liquid HMDSN is compared to that of the plasma deposited film: it is evident a marked decrease of the NH stretching at $1185 \mathrm{~cm}^{-1}$ (peak n. 1) and of the Si-NH-Si asymmetric stretching at $935 \mathrm{~cm}$ 1 (peak n. 2), as well as the sharp appearance of $\mathrm{CH}_{2}$ wagging band in $\mathrm{Si}-\left(\mathrm{CH}_{2}\right) \mathrm{n}$ $\mathrm{Si}$ at 1030 (peak n. 3), that of $\mathrm{Si}\left(\mathrm{CH}_{3}\right) \mathrm{n}$ at $800 \mathrm{~cm}^{-1}$ (peak n. 4), and that of $\mathrm{SiH}$ at $2140-2100 \mathrm{~cm}^{-1}$ (not shown in the figure). The overall effect of increasing oxygen concentration of the feed, in addition to the increase of monomer activation, is to decrease the hydrogen and carbon content of the film. This can occur by homogeneous gas phase reactions, which form new film precursors ( $P$ and $P^{\prime}$ ) and/or by etherogeneous gas-surface oxidations. Even if it has been reported on the basis of theoretical considerations that etherogenous gassurface oxidation is always the main channel of carbon removal (24), it is our 
opinion that, when the oxygen-to-monomer ratio of the plasma is low the major role is played by homogeneous reactions. The final products of oxidation are, in any case, not only $\mathrm{CO}, \mathrm{CO}_{2}$ and $\mathrm{H}_{2} \mathrm{O}$ but also partially oxidized organic compounds with reduced polymerizing capability $(18,19)$.
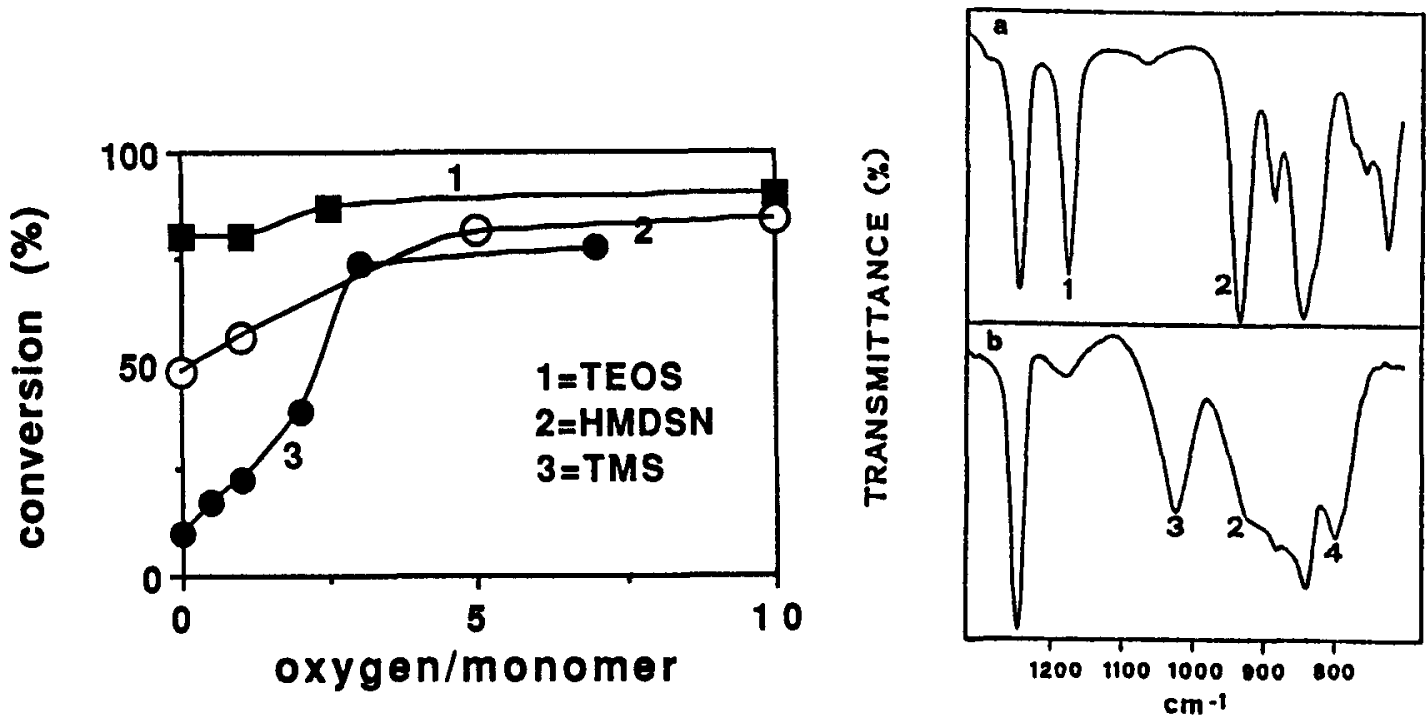

Fig. 5. Conversion detected by means of mass spectrometry for different monomer under different experimental conditions.

Fig. 6. FT-IR spectra of a) liquid Hexamethyldisilazane, b) thin film deposited in HexamethyldisilazaneAr plasma.

\section{REFERENCES}

1. D.M. Manos and D.L. Flamm, Plasma Etching: an introduction + Academic Press, New York (1989).

2. O. Auciello and D.L. Flamm, Plasma Diagnostics: Vol. 1, Academic Press, New York (1989).

3. R. d'Agostino, Plasma Deposition, Treatment, and Etching of Polymers, Academic Press, New York (1990).

4. G. Bruno, P. Capezzuto, and A. Madan, Plasma Deposition of Amorphous Silicon-Based Materials, Academic Press, New York, in press.

5. J.W. Coburn, IEEE Transaction on Plasma Science, 19, 1048 (1991).

6. M. C. Peignon, Ch. Cardinaud, and G. Turban, J. Electrochem. Soc.. 140, 512 (1993). 
7. R. d'Agostino, P. Capezzuto, F. Cramarossa, and F. Fracassi, Plasma Chem. Plasma Process., 9, 513 (1989).

8. F. Fracassi, J.W. Coburn, J. Appl. Phys., 63, 1758 (1988).

9. R. d'Agostino, F. Fracassi, C. Pacifico, and P. Capezzuto, J. Appl. Phys., 71, 462, (1992).

10. G. Turban, J.F. Coulon, N. Mutsukura, Thin Solid Films, 176, 289 (1989).

11. F. Fracassi, R. d'Agostino, work in preparation.

12. N. Couchman, C. Pacifico, G. Turban, B. Grolleau, Applied Surface Science, 70, 613 (1993).

13. R. d'Agostino, F. Fracassi, and C. Pacifico, J. Appl. Phys., 72, 4351, (1992).

14. J.W. Coburn, Pure and Appl. Chemistry, 64, 709 (1992).

15. C.P. Chang, C.S. Pai, J.J. Hsieh, J. Appl. Phys, 67, 2119 (1990).

16. C.S. Pai, C.P. Chang, J. Appl. Phys., 68, 793 (1990).

17. N. Selamoglu, J. A. Mucha, D.E. Ibbotson, D.L. Flamm, J. Vac. Sci Technol. B, 7, 1345 (1989).

18. F. Fracassi, R. d'Agostino, P. Favia, J. Electrochem Soc., 139, 2639 (1992).

19. C. Charles, P. Garcia, B. Grolleau, G. Turban, J. Vac. Sci. Technol. A, 10, 1407 (1992).

20. F. Fracassi, R. d'Agostino, P. Favia, work in preparation.

21. H. Yasuda, Plasma Polymerization, Academic Press, New York, (1985).

22. F. Fracassi, R. d'Agostino, P. Favia, M. van Sambeck, Plasma Sources Sci. Technol., (1993), in press.

23. P. Favia, R. Lamendola, R. d'Agostino, Plasma Sources Sci. Technol., 1, 59 (1992).

24. G.P. Raupp, T.S. Cale, J. Vac. Sci Technol. B」 10, 37 (1992). 